\title{
Chinese Dreams of Happiness: What Are the Chances?
}

\section{Gerda Wielander}

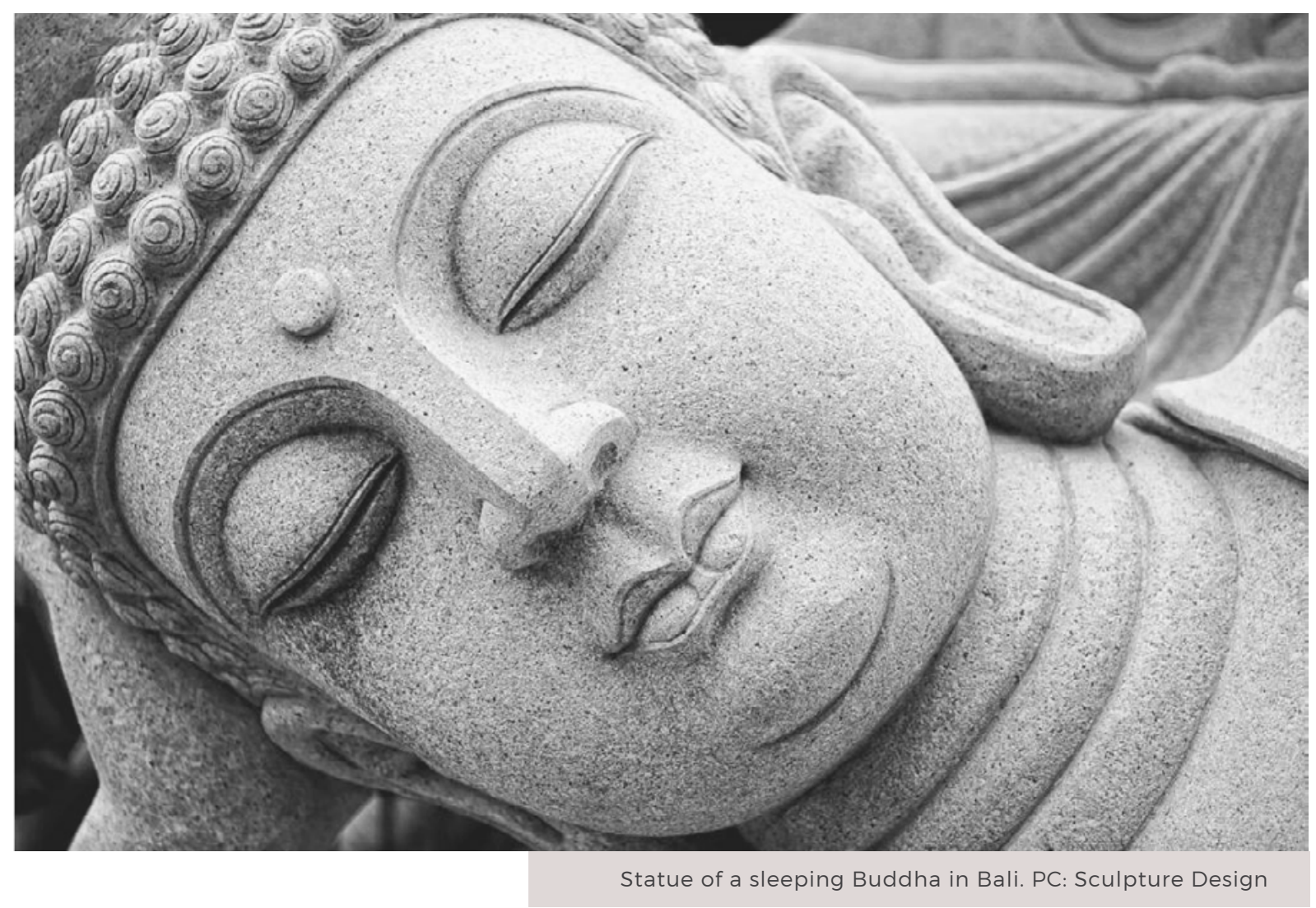

The happiness of the Chinese people is one of the declared aims of the Chinese dream. An educational volume on social governance and welfare published by the official Red Flag Press titled Building a Happy China sums it up like this: 'The process of the reinvigoration of the Chinese nation equates to the building of a happy China; without people's affluence, development cannot be considered successful; without people's happiness, the reinvigoration will not be complete' (Shu 2013, I). The happiness of the Chinese people is not just an aim, but has also become a measure for the realisation of the Chinese dream. Put differently, being happy is the required spiritual state of the Chinese people in this new project of the Party-state. But what methods are available to the Chinese people to achieve happiness? In the following essay, I will briefly look at three different options from the field of psychology and psychotherapy-psychodynamic therapies, positive psychology, and Morita therapy-to discuss the chances for fulfilment of China's dream of happiness.

\section{A Process of Collective Transformation}

Moving from a state of unhappiness to happiness requires a transformation at the 
individual level, which-provided that enough individuals go through the same processthen becomes a collective transformation. This is an evolutionary process guided by people's ability to adapt their strategies to maximise happiness depending on needs and opportunities. When needs shift for many people at the same time (due to massive changes in their socio-political environment, for example), then similar adaptation strategies will be adopted. These, in turn, accumulate into collective trends, which have the potential to lead to social change (Welzel and Inglehart 2010).

The marketisation of most, if not all, aspects of the Chinese economy and the liberalisation of society have brought into existence a wide range of potential role models and 'happiness maximisation strategies' for the Chinese individual to choose from. In a radically changed economic and social environment, people's readiness to choose and try out new strategies to maximise happiness has increased considerably. At the same time, today the government's attempts at re-affirming normative expectations of behaviour and the 'correct spirit' around the values of socialism compete with alternative value systems derived from religions and different schools of thoughts that have been circulating among the population. Various schools of psychology also play a role in this.

\section{The Limits of Freudian Psychoanalysis}

Western culture in the twentieth century has grown up on the pervasive influence of Freudian concepts of the unconscious and tends to focus on the importance of individual transformation in isolation from the social. Only very few psychoanalytical schools offer perspectives on transformation that go beyond the individual. Otto Gross (18771920), an Austrian psychoanalyst, could not conceive of transforming the self without the transformation of the socio-political context; to him factors outside the individual's history needed to be taken into account in an individual's unhappiness (Heuer 2003). More recently, the British Jungian analyst Andrew Samuels has argued that the inner world of emotion and fantasy about survival and status builds up in a ceaseless feedback loop with the outer world; and outer world problems in society gain emotional and fantasy elements within the individual (Samuels 2014). Hence-according to him-the economic (and political) and the psychological mutually irradiate.

Freudian ideas of the unconsciousintroduced to China in the 1920s-with their focus on sexual trauma never really suited Chinese culture, and interest in its application to the Chinese mind was limited, despite a brief 'Freud fever' in the 1980s. But, although the soul or the unconscious have never played a significant role in the Chinese concept of psychological transformation, China's transformation into a 'happy and prosperous socialist society'-a slogan used in the 1950s-always required the transformation of both the individual and the external circumstances. The overthrow of the existing social order or the redistribution of means of production alone were never considered enough to achieve happiness even in socialist China.

Psychodynamic therapies have developed from Freudian and Jungian ideas, and in essence are based on an engagement with one's individual past and on working through trauma through verbal exchanges, often over a long period of time. It is an individualistic, non-directive, backward-looking process, which is painful, costly, and while often successful, results don't appear quickly. It requires a willingness to critically engage and often break with one's past before being able to move forward. Considering how important the tight control of all interpretations of China's past is for the legitimacy of the ruling power and promoter of the Chinese dream, 

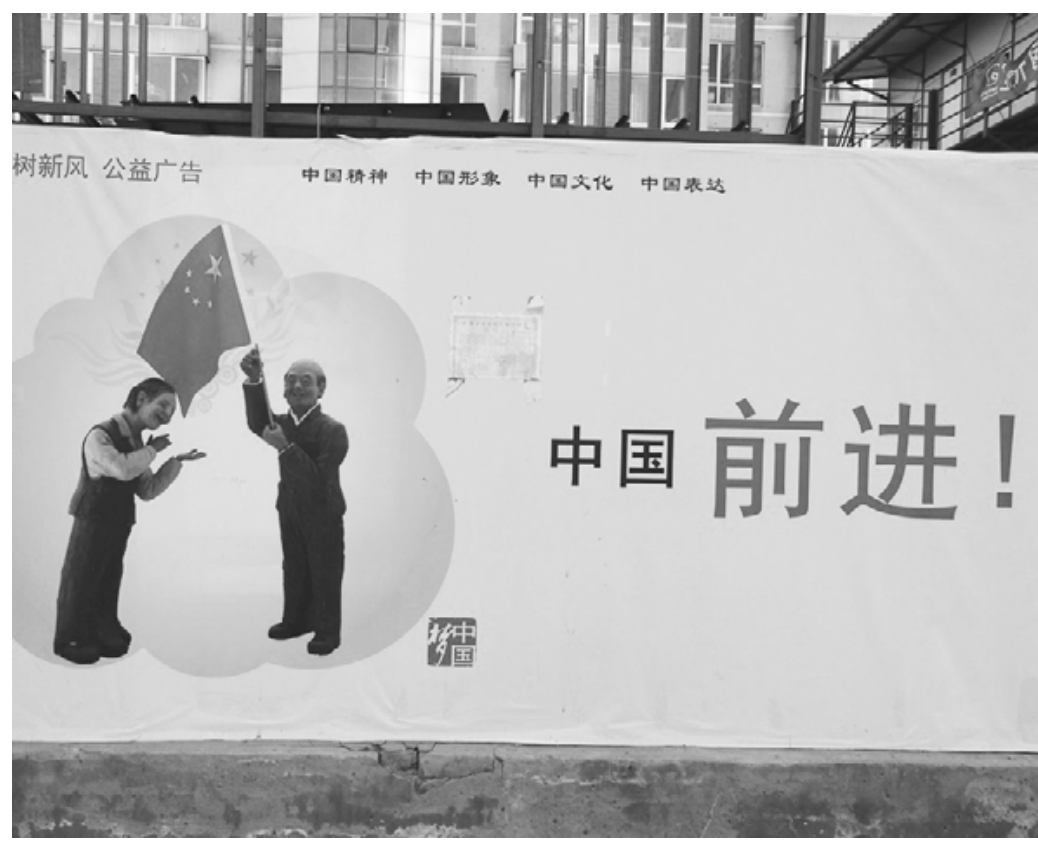

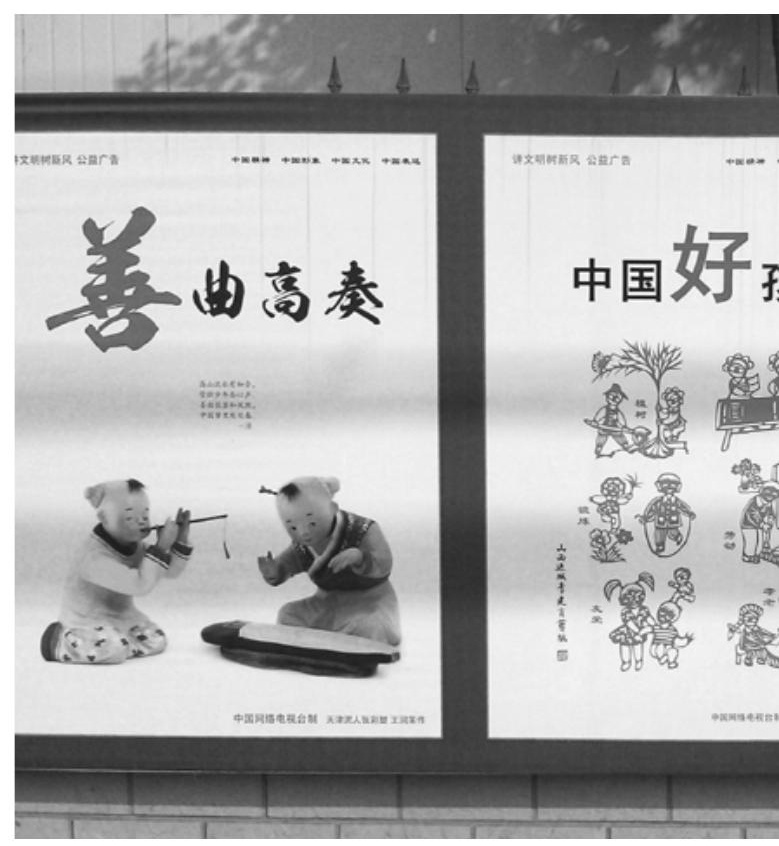

the chances or even possibility of gaining closure, and hence happiness, by working through past traumas are nil.

\section{The Communist Party's Love Affair with Positive Psychology}

The Chinese Communist Party (CCP) is, however, very much enamoured with positive psychology, a field of psychology that developed out of the behaviourist school, which focuses on 'flourishing', that is psychological functioning at its best. The central premise is that too much of psychology has been focusing on negativity, complexes, depression, and other shortcomings, while the needs of 'normal people' (not usually put in quotation marks in the Chinese literature) and the myriads of beautiful and positive things in the world have been neglected.

Positive psychology has developed out of humanistic psychology, which, in turn, responded to the limitations of psychodynamic approaches built on Freudian psychoanalysis. The philosophical foundations of humanistic psychology lie in existential philosophy, with its concern for human freedom, choice and responsibility, and search for meaning. However, despite this shared philosophical tradition there are fundamental differences between humanistic and positive psychology in relation to freedom, will, agency, personal responsibility, and determinism on the one hand, and the nature of phenomenological experience on the other. Existentialist philosophy holds that there are no absolute standards or criteria that can be used when making life decisions. Existentialists view experience as something inherently individual and thus inter-subjectivity is a problem that can be only partially resolved. Existential philosophy-and humanistic psychologyconsider communication, by necessity, unreliable. No existentialist philosopherand no humanistic psychologist-will tell an individual what to do (Waterman 2013).

Positive psychology, on the other hand, 
'scientific outlook on development' (kexue fazhan guan), which is a key aspect of contemporary Chinese socialist ideology. In terms of efficacy, positive psychology, like many other forms of cognitive behavioural therapy, can have great results in the short term, but do not tend to have a long-lasting effect. As such, China may be able to hit its happiness targets in the short run, but should expect a magnified wave of unhappiness to return in the mid-term (Burkeman 2016).

\section{Beyond Mind Control: Morita Shoma and Acceptance}

One of the main reasons why positive psychology does not help in the long run is that it does not provide any answers to the profound existential questions about the meaning of life, moral value, and the question of transcendence, which so many Chinese individuals are now contemplating as their livelihoods have been improved and middleclass anxieties are growing. Nor is positive psychology interested in individual (hi) stories or journeys and in-depth dialogue or engagement with individuals in the way psychodynamic or humanistic therapies and some religious practices are.

Xi Jinping pays great lip service to the importance of China's indigenous religions when it comes to looking after the spiritual needs of the Chinese people. He may therefore be open to the approach taken by Morita therapy, which was developed in the first half of the twentieth century by a man called Morita Shoma. Building on Western psychiatric medicine without the influence of Freudian psychoanalytic concepts, and drawing on the concepts and practices of Zen Buddhism, Morita therapy is not concerned with technique or procedure, but with the enhancement of a philosophical view that is strongly rooted in the Buddhist and Daoist views of life, and of the relationship between mankind and nature (Kenji 2005).

Morita therapy focuses on present circumstance and does not believe in the value of digging into the past. Morita Shoma saw no therapeutic meaning in searching and bringing to consciousness certain traumatic incidents. On the contrary, in his view, the source of unhappiness lies in the nature of one's disposition and in an excessively acute awareness of the reactions of one's own mind and body-something that is of course encouraged, almost celebrated, in psychodynamic approaches. But Morita therapy also contradicts positive psychology or any other attempts to control and use our minds to relieve anxiety. Positive psychology shuns negative emotions and focuses the individual's energy to change or avoid them, but in Morita therapy negative emotions are simply part of nature and not problematic; it is the decision to view them as negative and undesirable which creates the problem. By deciding that a certain emotional state is somehow negative in the context of one's life, too much focus is placed on the reaction to the negative emotion, which in turn intensifies the emotional reaction, attracting the person's attention to it even more. A destructive circle of obsession is thus created.

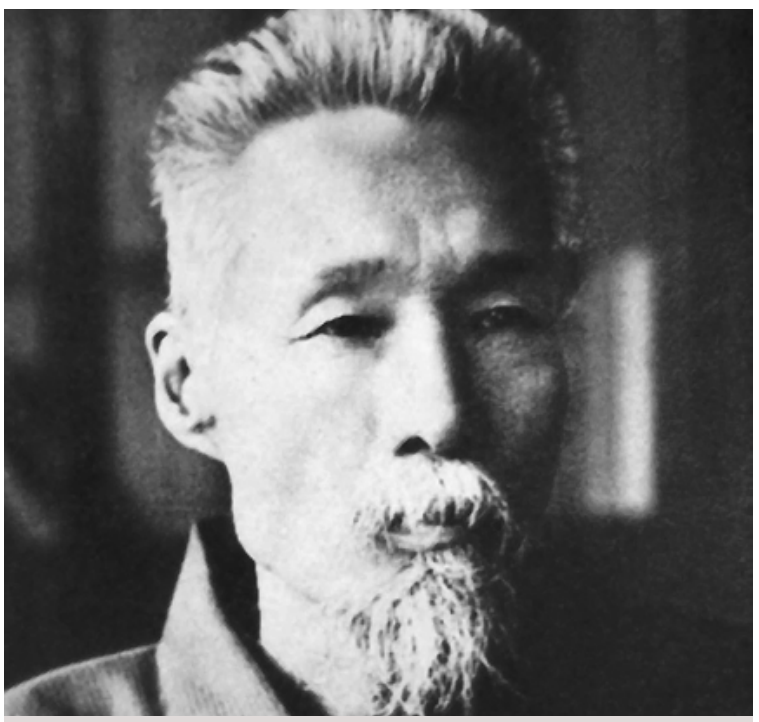

A photo portrait of Morita Shoma in old age PC: Wikipedia. 
The aim of Morita therapy is not to identify the cause of unhappiness, but the destruction of this vicious circle, which creates this obsession with negative emotion. Morita therapy strives for harmony between nature and ideas-where nature includes all emotions, desires and bodily feelings, and ideas are all theories substantiated by means of language. According to Morita therapy it is counter-productive to try to control one's emotions. The starting point in the healing process is acceptance of suffering, its goal an awareness of the causes-always multipleof suffering, without seeking to control the suffering.

The ideal state of mind in Morita therapy is that of arugamama, which is the ability to accept things as they are:

When we can accept reality, then we become more obedient. In reality, there is no such thing as making great strides. To think that one suddenly becomes enlightened and becomes a better person is not based on object reality. It is an assessment based on moods. One becomes great by taking one step at a time. One should not take it for granted that one has become great. (Kenji 2005, 179)

\section{Pursuing Utopian Dreams}

Aspirational, utopian dreams rarely improve people's lives, including their psychological lives. The German Chancellor Helmut Schmidt (1918-2015) famously advised: 'Anyone who has visions should go to the doctor.' Angela Merkel-who lived through thirty years of socialist utopia-and her Northern European counterparts are all famously down to earth and pragmatic, and yet their countries lead the world's happiness rankings. So, to paraphrase Morita, and to return to the development targets of the Chinese dream, one should not take it for granted that one will become happy. The first obstacle on the road to China's happiness is the articulation of the Chinese dream itself. Giving up the obsession with the dream will be the first step towards its fulfilment.

\section{Gerda Wielander}

Gerda Wielander is Associate Professor in Chinese and Head of Modern Languages and Cultures at the University of Westminster, London. Her main research interest lies in the link of the personal and spiritual to wider social and political developments in modern and contemporary China. She is the author of Christian Values in Communist China (Routledge 2013) as well as several book chapters and articles in leading peerreviewed journals. She is currently working on an edited volume called Perspectives on Chinese Happiness, which is part of her wider research on happiness targets and education in China's political project. 
This text is taken from Disturbances in Heaven: A Year of Chinese Labour, Civil Society, and Rights, Made in China Yearbook 2016, edited by Ivan Franceschini, Kevin Lin and Nicholas Loubere, published 2017 by ANU Press, The Australian National University, Canberra, Australia. 